\title{
The perception of assimilation and brightness contrast as derived from code theory
}

\author{
EMANUEL LEEUWENBERG \\ University of Nijmegen, Nijmegen, The Netherlands
}

\begin{abstract}
It is shown that assimilation and brightness contrast effects are evoked by structural aspects of patterns. In a pilot experiment, variously shaped gray patterns were used as stimuli. The backgrounds used with each of these shapes were identical: half black and half white. If the gray area against the black part was judged to be more black than the gray area against the white part, an assimilation effect will have occurred; when the reverse occurred, this was called a contrast effect. The task was to rank-order the stimuli on the assimilation-contrast scale. It is argued that the two effects are due to two different interpretations, each derivable from a different code of a pattern. The simpler the contrast code is with respect to the assimilation code, the more it will be perceptually preferred. In the specification of pattern complexity, structural information theory was used. A significant correlation was discovered between the theoretical preference for the contrast interpretation and the contrast preference of subjects.
\end{abstract}

Both assimilation and contrast phenomena are illusory effects. The first occurs if some element is judged as tending toward the surrounding, and the second occurs if some element is judged as being more opposed to the surrounding. In this sense, assimilation and contrast are opposing effects. The two effects have been demonstrated in several studies, for example, ones on social relations and attitudes (Sherif \& Hovland, 1961), on perceived size (Goldmeier, 1972), and on perceived distances of line lengths, including the Müller-Lyer illusions (Restle \& Decker, 1977). Remarkable assimilation illustrations using colored arabesque patterns were given by Bezold (1874). Such demonstrations gave rise to experiments on assimilation and brightness contrast (Helson, 1963; Helson \& Joy, 1962). When the width of black-white lines on a gray background was varied, it was found that assimilation diminished and contrast in the gray background increased with the width of the lines. In Figure 1, the gray parts in the left pattern demonstrate assimilation, and those in the right pattern, contrast effects.

Helson proposed that there was a continuous dimension from assimilation to contrast: the more of the first, the less of the last. In this paper, this assumption is considered as a point of departure. The lack of contrast is due to assimilation. Helson's results are commonly explained in physiological terms, such as summation and inhibition. In this paper, an attempt is made to study the assimilation and contrast effects for various shapes, with all having the

The author's mailing address is: Department of Experimental Psychology, University of Nijmegen, P.O. Box 9104, $6500 \mathrm{HE}$ Nijmegen, The Netherlands. same depth of gray and the same surface area and all being displayed against a white/black background. In other words, sensory aspects such as size and brightness are kept constant, while only the shape of the areas is varied. Here, the level of explanation tends to be more cognitive and is expressed in terms of the "structural information" of the shapes. Later in the paper, structural information theory (Buffart, Leeuwenberg, \& Restle, 1981; Leeuwenberg, 1971) will be briefly summarized in order to clarify how the so-called structural information (I) of patterns will be used as a measure of complexity and will be applied to the assimilation and contrast data of the experiment described below. First, some general points of structural information theory will be presented as an introduction to the analysis of the assimilation and contrast phenomena.

\section{Complementarity and Completion}

The pattern of Figure 2 can be interpreted as a mosaic of parts, an $L$ shape and a square, or as a pair of squares, one occluding the other. The code of the last interpretation (see below) has less structural information $(I=4)$ than the code of the first $(I=6)$. According to structural information theory, the last interpretation is perceptually preferred. The relative preference $(\mathrm{P})$ of the minimum interpretation is:

$$
P_{(\min )}=6 / 4 \text {. }
$$

Besides the mosaic interpretation, there are, however, more alternative interpretations of the minimum interpretation. Indeed, each subdivision of the pattern refers to a specific interpretation. However, these are all more complex than the mosaic interpretation. Furthermore, the last interpretation is to some 

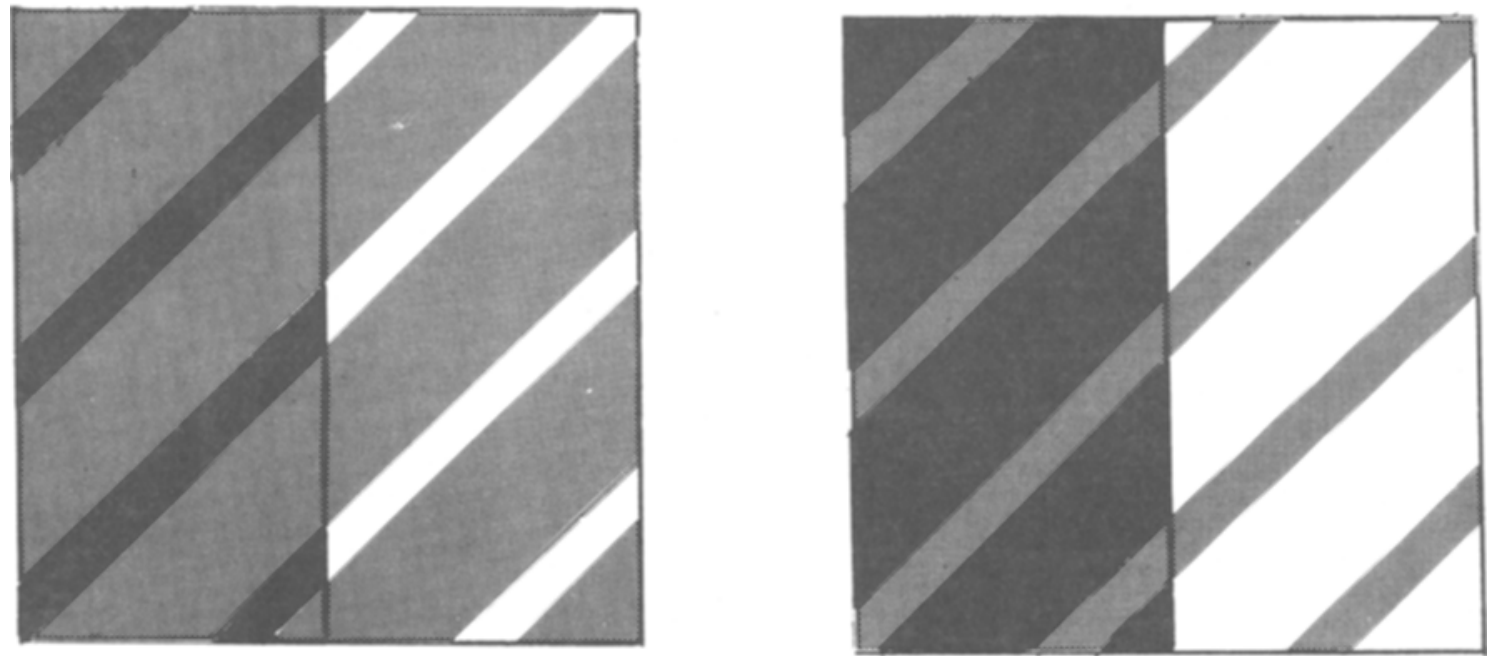

Figure 1. The left pattern shows more assimilation; the right pattern shows more contrast effect.

extent opposed to and yet complementary to the minimum interpretation (Van Tuijl \& Leeuwenberg, 1979). What does this mean? The minimum code allows all pairs of squares in any relative position. Thus, this code is incomplete. The same holds for the mosaic code: the positions of the $L$ shape and the square are not fixed. However, the combination of the mosaic and the minimum interpretation fixates the pattern of Figure 2. This point is further elaborated by Leeuwenberg and Buffart (1982).

In summary, two interpretations are involved in the specification of a preference measure, the minimum interpretation and the simplest complementary interpretation.

Before going into the assimilation and contrast phenomenon, a second point may be worthwhile to keep in mind. In Figure 2, a part of the background square is invisible, being occluded by the foreground. Nevertheless, this foreground-background interpre-

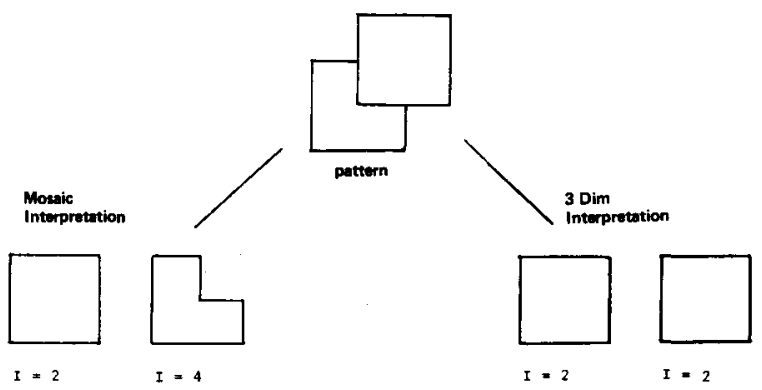

Figure 2. This pattern can be interpreted as a mosalc or as two overlapping squares. tation is perceptually preferred. This agrees with the "minimum principle," being part of structural information theory. In Figure 3, a similar case is presented. The pattern can be interpreted as three disjunct parts $(I=9)$, but also as a cross with three gaps $(I=7)$. The main part of the last interpretation is the cross. The gaps play a more secondary and supplementary role (Leeuwenberg, 1978). The cross interpretation will hold as long as there are not too many gaps. The regularity of the cross pattern makes it worthwhile to overlook the gaps. These distortive gaps, which have to be completed, are considered here as "supplementary components."

\section{Assimilation (A) and Contrast (C)}

Figure 4 shows a pattern that was used in the experiment. The other experimental patterns showed similar constraints: The surface of the gray part is kept constant and is divided into two equal parts by the middle line. For all the patterns, the background is equal: half black and half white. The main variable is the shape of the gray pattern and the orientation with respect to the background middle line. For the moment, therefore, the background will be disregarded.

Let us focus on Figure 4. The gray part, conceived of as one single pattern, is a square (Figure 5a). However, the code of the square describes any square in any orientation. The gray surface may also be conceived of as being two parts, separated by the middle line (Figure 5b). However, the code of these parts allows any relative position between these parts. If both codes refer to one shape, the gray pattern in Figure 4 is fixed, except for the $y$-coordinate 


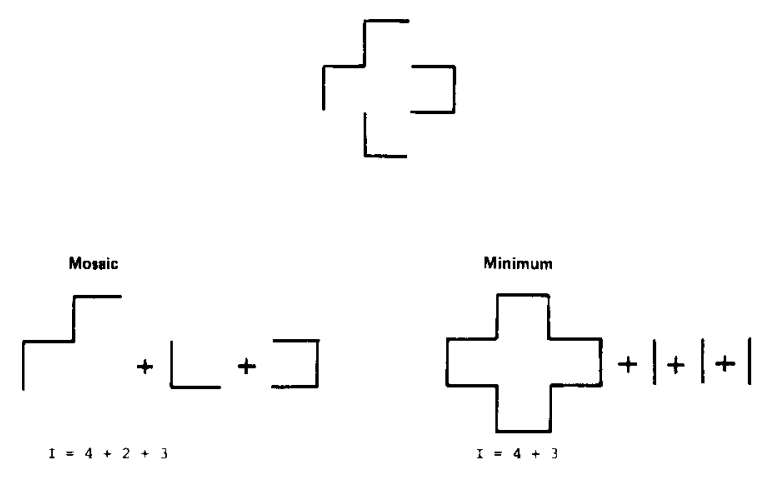

Figure 3. This pattern can be interpreted as a set of parts, or as a cross with gaps.

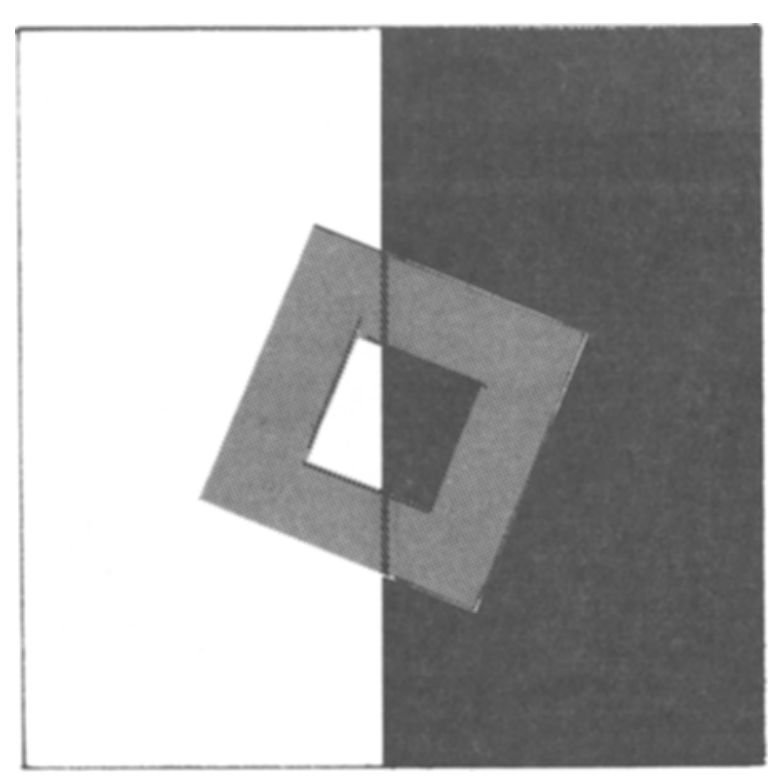

Figure 4. One of the 20 experimental patterns.

(this will be disregarded because it applies for both codes). The first code of the total pattern fixates the relative position of the parts described by the second code. The last code fixates the orientation of the first code. In this sense, both codes are complementary.

Now the way in which the first code is related to assimilation and the second code to contrast will be demonstrated, using a simpler pattern, presented in Figure 6.

The numbers in the pattern refer to the degree of darkness. The rectangle, or pair of squares, is gray (2) and the background is half dark (3) and half "white" (1). For this pattern, the A effect occurs when the left gray area on the dark background is seen as darker than the right gray area on the white background. The contrast effect occurs when the left gray area is seen as lighter than the right gray part.
According to our point of view, these two effects are evoked by two different interpretations, each derived from two different decompositions of the pattern. Analogously to the vectorial analysis of color according to Beck (1966) and Metelli (1974), a pattern is regarded as a composite of different, superimposed transparent light configurations or, more concretely, as the result of different slides projected onto one screen.

The assimilation interpretation comprises the following components: a white (1) frame (see Figure 6b), superimposed on which is a bright $(-1)$ rectangle
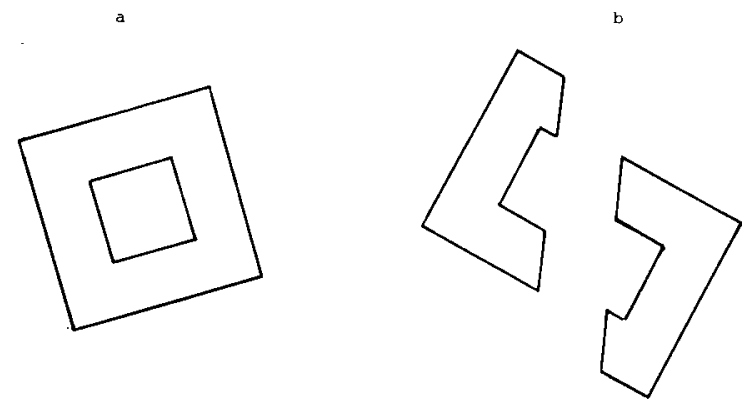

Figure 5. The two simplest organizations of the gray part of Figure 4.

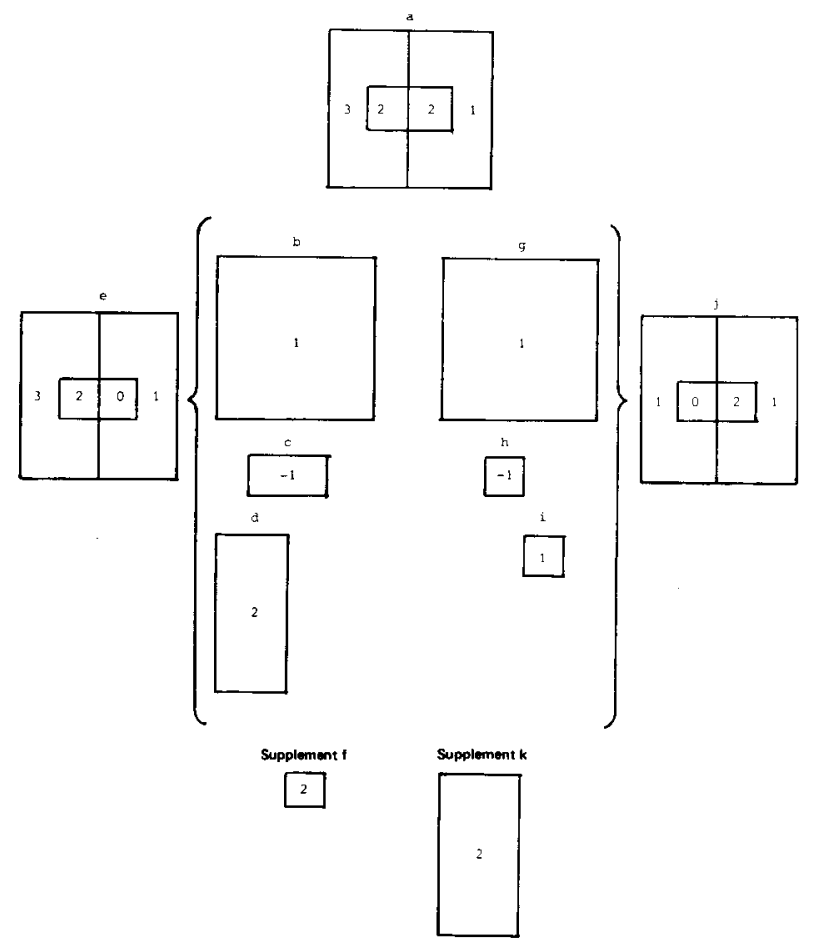

Figure 6. Two decompositions of the above pattern are presented. The components on the left side refer to the assimilation interpretation; those on the right refer to the contrast interpretation. 
(Figure 6c) and, over this, a shadow (2) projection at the left side of the frame (Figure $6 \mathrm{~d}$ ). The resulting composition, shown in Figure 6e, reflects the assimilation interpretation: the left half (2) of the rectangle is darker than the right half (0). A supplementary projection (Figure 6f) would be required on the right half of the rectangle in order to arrive at the above stimulus pattern $a$.

The contrast interpretation breaks down into the following components: a white (1) frame (Figure 6g), upon which is superimposed a bright $(-1)$ square (Figure 6h) and an adjacent less bright (1) square (Figure 6i). The resulting composition, shown in Figure $6 j$, reflects the contrast interpretation: the left square is brighter than the right square. Here, too, a supplementary projection (Figure $6 \mathrm{k}$ ) would be réquired to generate pattern $a$.

There is also a third decomposition to be considered, one consisting of three mosaic components: the solid black part, the white part, and the total gray part. The black part and the white part show holes left over for the gray rectangle. It can easily be shown that this mosaic composition is always more complex than the assimilation and contrast decomposition. It is generally reasoned that the contours of the gray shape are presented in a positive and a negative way. These contours are thus represented twice; that is, they are redundant. For this reason, the mosaic interpretation will not play a role in the analysis of the patterns.

Our hypothesis assumes that the least complex composition will be perceptually preferred. Because the data of the experiment are tested at ordinal level, the predictive preference measure will be simplified. The components common to both assimilation and contrast are disregarded. However, the order thus simplified is equal to the order of the complete preference measures. Those components with the same contour complexity are regarded as equal (see below). Thus, the frames (Figures $6 \mathrm{~b}$ and $6 \mathrm{~g}$ ), the big rectangles (Figures $6 \mathrm{~d}$ and $6 \mathrm{k}$ ), and the little squares (Figures $6 f$ and 6i) cancel each other out. The remaining components are the rectangle (Figure 6c) and the little square (Figure 6h). The greater the complexity (I) of the assimilation component, the rectangle, and the less the complexity of the contrast component, the little square, the greater is the preference $(P)$ for the contrast interpretation. The $I$ of the rectangle is 3 and that of the square is 2 (see below). Thus,

$$
P(C)=I(A) / I(C)=3 / 2 \text {. }
$$

The same analysis may be applied to the second pattern shown in Figure $7 \mathrm{~b}$. The crucial assimilation component here is also a rectangle $(I=3)$, whereas the shape representing the contrast interpretation is now more complex $(I=5$, see below). The preference

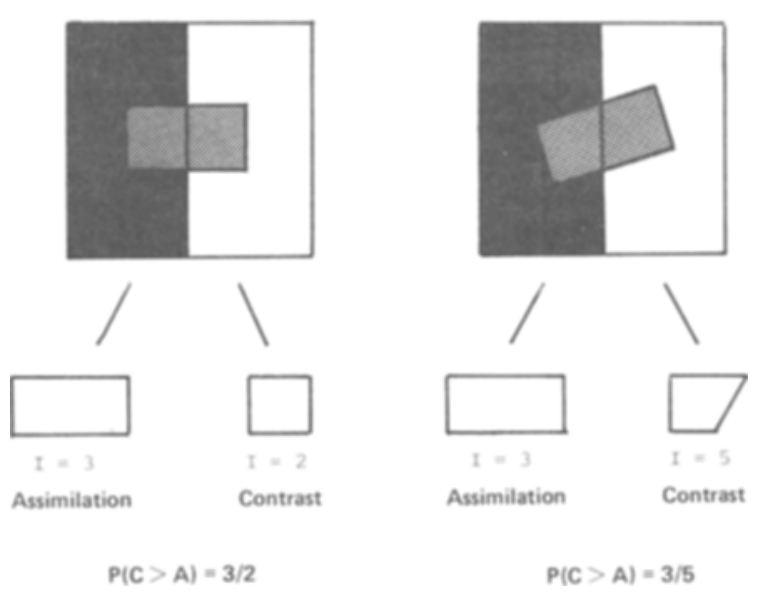

Figure 7. According to assimilation and contrast, the background codetermines the preferred interpretation. The left pattern is in favor of the contrast; the right pattern is in favor of the assimilation interpretation.

(P) for the contrast interpretation of the pattern in Figure $7 b$,

$$
\mathrm{P}(\mathrm{C})=\mathrm{I}(\mathrm{A}) / \mathrm{I}(\mathrm{C})=3 / 5,
$$

is lower than the contrast preference of pattern a in Figure 7. The gray area in Figure $7 a$ is in a stronger relationship to the background borderline. Hence, in this case, the gray area is more easily subdivided in two equal squares symmetrically related with respect to the middle line. In general, contrast will be seen if some gray part that is surrounded by white and another gray part that is surrounded by black can easily be conceived of as a gray figure standing out against a white background and another separate gray part as another distinct figure on a black background. As a consequence, the black middle line will enhance the contrast effect. Note that, for the sake of simplicity, this black middle line is not specified in the codes, while for all experimental patterns it will favor the preference for contrast to the same extent.

In Figure $7 \mathrm{~b}$, each of the two parts is more complex than the total rectangle, due to its oblique orientation. It is therefore more difficult to perceive two separate parts, each standing out as a distinct figure against each background. Consequently, the probability that these two parts have different surface colors is also decreased. The gray part has here one single shape and, therefore, one surface color. Any effect of different colors is due to the surrounding inasmuch as the gray part shares a common fate with this surrounding.

In summary, the two simplest decompositions of the above patterns imply assimilation and contrast 
effects. The first is facilitated if the total configuration is simpler, and the second, if the local pattern is simpler. While other decompositions are more complex, the assimilation and contrast effects are considered as complementary, although they are evoked by qualitatively different interpretations. The preference measure is here simplified, while only ordinal predictions are relevant for the experiment. This measure is to some extent in favor of the assimilation interpretation, since the middle line, present in all experimental patterns, is not specified in the codes.

The above analysis will be applied equally to all experimental patterns. The preference measure is specified by the information contained in the total gray pattern and that in the half pattern. In the following section, structural information theory will be introduced in order to quantify perceptual complexity.

\section{Coding System}

As a measure of "complexity," we propose the employment of structural information as represented by a pattern code. Use is to be made of a previously developed coding system which has already been discussed in detail elsewhere (Buffart, Leeuwenberg, \& Restle, 1981; Leeuwenberg, 1969, 1971; Van Tuijl, Leeuwenberg, 1979).

In the following paragraphs, the technique for generating the pattern code, rather than the theory of the code itself, will be described briefly. It should be noted that neither the code theory nor the technique refer to the actual process of information input, but only to the final result of that process, that is, to the description of patterns.

In the system under discussion, the contour of a pattern is initially described by the successive lengths of straight line elements $(1, \mathrm{~m}, \mathrm{n}$, etc.) and the angles ( $a, b, c$, etc.) subtended between them. This series of line lengths and angles describing the contour is referred to as the "primitive code."

In a following phase, a "final code" is constructed by abbreviating the primitive code. This is achieved by the stepwise performance of a number of specific reduction operations. At each step, the choice of the next operation must be such as to reduce the code as much as possible. When a stage is reached at which no operation is able to reduce the code further, the result is called a "final code." The number of operations employed, together with the remaining irreducible elements in the code, is conceived of as a measure of "structural information." In every complex pattern, there are various (at least two) ways to proceed from a certain primitive code to one or another final code. These different routes do not, of course, necessarily involve the same number of steps. The various final codes derivable from a single primitive code therefore reflect different measures of structural in-
Table 1

The Four Admissible Reduction Operations as Applied to Six Selected Primitive Codes

\begin{tabular}{|c|c|c|c|}
\hline Operation & $\begin{array}{c}\text { Primitive } \\
\text { Code }\end{array}$ & Final Code & I \\
\hline \multirow[t]{2}{*}{ (1) Iteration } & $\mathrm{a} a \mathrm{~b} b$ & $2 *[(a)(b)]$ & 3 \\
\hline & $a b a b$ & $2 *[(a \mathbf{b})]$ & 3 \\
\hline (2) Continuation & a a $\mathbf{a} \ldots$ & $\rho ;(a)$ & 1 \\
\hline \multirow[t]{2}{*}{ (3) Symmetry } & $a b b a$ & Sym [a b] & 3 \\
\hline & $\mathrm{a} b \mathrm{c} b \mathrm{a}$ & Sym $[a b(c)]$ & 4 \\
\hline (4) Distribution & $a b c b$ & $\langle(\mathrm{a})(\mathrm{c})\rangle \mid\langle(\mathrm{b})\rangle$ & 3 \\
\hline
\end{tabular}

Note $-I=$ structural information.

formation. The final code that possesses the least "structural information" is called the "minimum code." The fundamental idea underlying the coding theory, as well as those predictions of perceptual phenomena to which it is applied, is that of the minimum principle. This states that the perceptually preferred interpretation is always that based on the minimum amount of structural information and, as such, always reflects the minimum code of the pattern perceived.

The only four admissible reduction operations, applied to six selected primitive codes, are presented in Table 1. In this synopsis, which also gives the assessed values of structural information (I), the letters $a, b$, and $c$ may stand for line lengths or angles or both.

\section{Comments on the Operations}

(1) The most common source of redundancy is "iteration." No justification will be given here for the assessment of the structural information value (I), which is indicated merely by the number of dots beneath the relevant symbol elements in the final codes.

(2) It may be that the cessation of a series of elements (a) stands in no need of prior specification because information about its termination is present elsewhere, as when the series halts upon meeting an already specified line or angle. Illustrations will clarify this rule later on.

(3) Any symmetry can be represented by means of this operation.

(4) In those cases in which some element (b) is regularly interwoven with other elements (ac), the periodic alternation can be represented by the given final code.

As indicated above, the primitive code is a description of the contour of a pattern. In the figures, orientation is indicated by an arrow and the start by a small circle. It should be noticed that the point of 
departure and the starting direction are open to choice, but that not every primitive code leads to the minimum code.

\section{Applications}

Starting with the simple pattern shown in Figure 8a, we shall construct its minimum code:

$$
\begin{aligned}
& \text { primitive code: la la la la } \\
& \text { continuation: } Q ;(1 \text { a }) \\
& I=2
\end{aligned}
$$

The last is the minimum code. The commencement of the primitive code here occurs arbitrarily at the bottom left corner, as indicated by the little circle. The combination of length 1 and angle $a=90$ continues until re-encountering the starting point: no extra information is required to indicate where the series halts, and hence the continuation operation is sufficient. The structural information value $=2$ is indicated by dots below the last final code.

Figure 8b can be coded as follows:

$$
\begin{aligned}
& \text { primitive code: } 1 \text { a m a } 1 \text { a m a } \\
& \text { continuation: } \quad Q ;(1 \mathrm{a} \mathrm{m} \mathrm{a)} \\
& \text { distribution: } \quad Q ;(<(l)(m)>\backslash<(a)>) \\
& I=3
\end{aligned}
$$

Figure $8 c$ can be coded as follows:

$$
\begin{array}{ll}
\text { primitive code: } & \text { a a l a m be ; (o) } \\
\text { iteration: } & 2 *[(1 \mathrm{a})] \mathrm{m} \text { be } ;(0) \\
\mathrm{I}=5 &
\end{array}
$$

Figure 8d can be coded as follows:

primitive code: m a m a la l a m a ma

continuation: $\varrho ;($ a ma 1 a la $)$

distribution: $\quad \mathrm{Q} ;(\langle(\mathrm{m})(\mathrm{m})(\mathrm{l})(\mathrm{l})\rangle \backslash\langle(\mathrm{a})\rangle)$

iteration: $\quad Q ;(<2 *[((\mathrm{~m}))((\mathrm{l}))]\rangle \backslash\langle(\mathrm{a})\rangle)$

$I=4$

It is easy to see that any other starting point would lead to a final code having more than four information units. This is because of an extra change in the angles involved in any other sequence.

Elsewhere (Buffart \& Leeuwenberg, 1982), the coding of holes in patterns is described more exten-

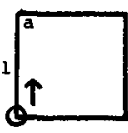

a

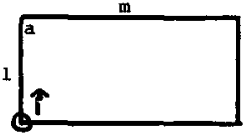

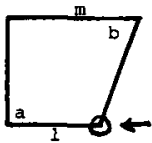

c

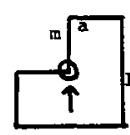

Figure 8. Patterns for which Illustrative codes are elaborated.

sively. It is also argued that a pattern code always describes a contour of a colored surface.

In the following section, a description is given of how assimilation and contrast judgments are derived for several different patterns. The data of this simple pilot experiment are further supplemented by predictions based on preference measures expressed in structural information loads.

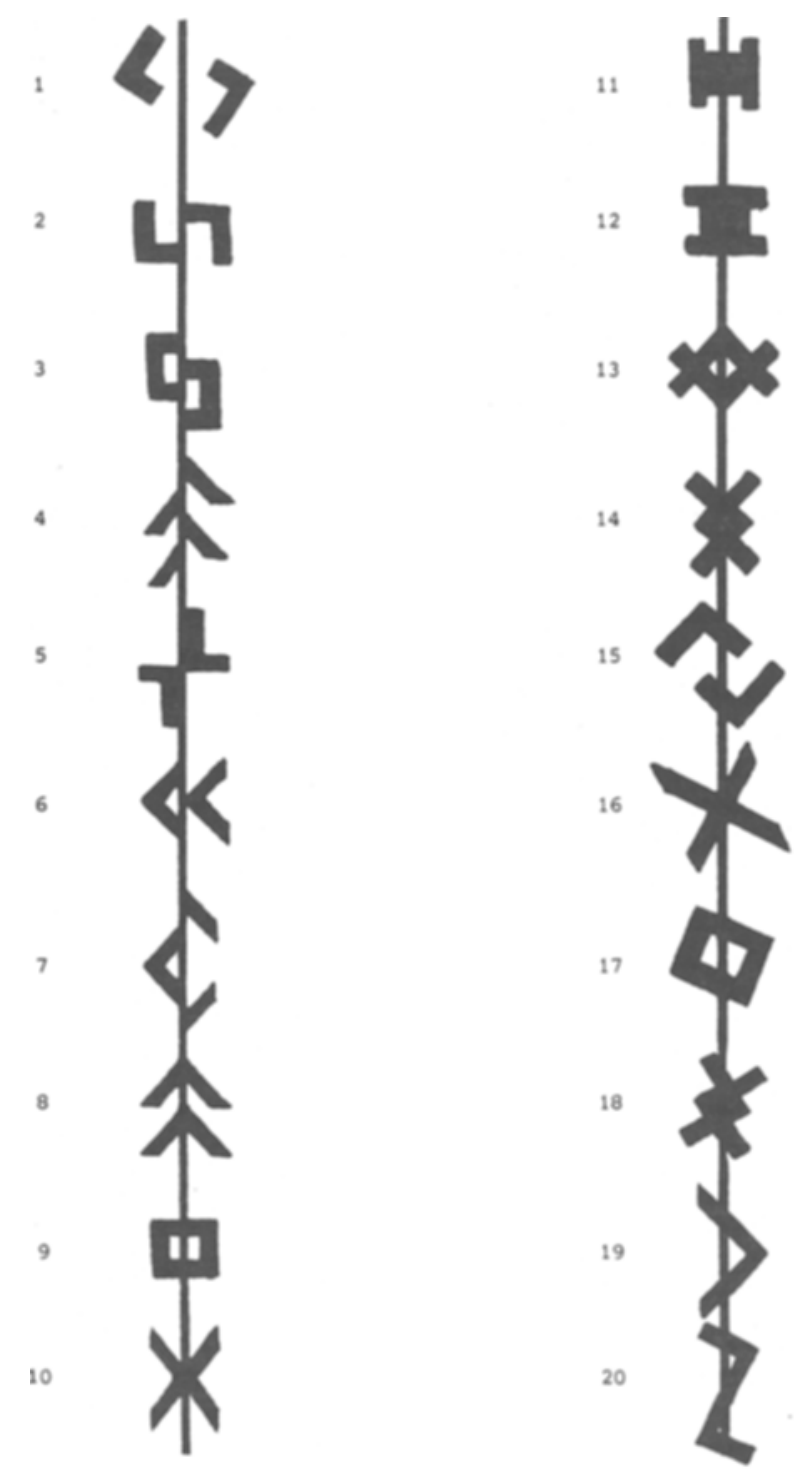

Figure 9. The gray :

iental stimuli.

Figure 9. The gray shapes of the experimental stimuli. 
Table 1

\begin{tabular}{ccccc}
\hline Column 1 & Column 2 & Column 3 & Column 4 & Column 5 \\
\hline 1 & $7 / 5$ & 19,5 & 529 & 20 \\
2 & $7 / 5$ & 19,5 & 521 & 19 \\
3 & $8 / 6$ & 18 & 460 & 17 \\
4 & $9 / 7$ & 17 & 457 & 16 \\
5 & $6 / 5$ & 16 & 443 & 15 \\
6 & $8 / 7$ & 14 & 499 & 18 \\
7 & $16 / 14$ & 14 & 440 & 14 \\
8 & $8 / 7$ & 14 & 233 & 8 \\
9 & $6 / 6$ & 10,5 & 417 & 13 \\
10 & $7 / 7$ & 10,5 & 290 & 12 \\
11 & $6 / 6$ & $10 / 5$ & 255 & 9 \\
12 & $6 / 6$ & $10 / 5$ & 218 & 7 \\
13 & $12 / 13$ & 8 & 265 & 10 \\
14 & $6 / 8$ & 7 & 145 & 2 \\
15 & $7 / 10$ & 5,5 & 216 & 6 \\
16 & $7 / 10$ & 5,5 & 146 & 3 \\
17 & $6 / 9$ & 3,5 & 275 & 11 \\
18 & $6 / 9$ & 3,5 & 85 & 1 \\
19 & $9 / 14$ & 2 & 215 & 5 \\
20 & $6 / 15$ & 1 & 198 & 4 \\
\hline
\end{tabular}

Note-In Column 1, the various gray patterns presented in Figure 9 are indicated. The preference measures for contrast are presented in Column 2. In Column 3, these are rank-ordered. In Column 4, the sum of the ranks (30 subjects) are presented. In Column 5, these are rank-ordered.

\section{EXPERIMENT}

\section{Material}

In the experiment, 20 stimuli $(10 \times 10 \mathrm{~cm}$ paper $)$ were used. The background of each stimulus was half black and half white (see Figure 4). Each stimulus employed a different gray pattern (see Figure 9). The surface area of all gray patterns was the same $\left(12 \mathrm{~cm}^{2}\right)$. As shown in Figure 4, the gray patterns were divided into two parts of equal surface area by a black line $(.5 \mathrm{~mm}$ width).

\section{Procedure}

The set of 20 stimuli was given to 30 subjects individually. In each set, the initial order of the stimuli was randomized. The task was to rank-order, without ties, the set of 20 stimuli according to strength of contrast effect. No time limit was set on reaching a decision, and the subjects were free to correct themselves.

\section{RESULTS}

In column 1 of Table 1 the patterns of Figure 9 are indicated. In column 2 the theoretical preference measures for the contrast interpretation are shown. In column 3, the ranks of these preference measures are presented. In column 4, the sum of ranks of contrast are given for the $\mathbf{3 0}$ subjects. In column 5 , these sums are rank-ordered. The Spearman rank correlation between columns 3 and 5 is: $r=.85$. The concordance (W) between subjects' judgments is significant $(p<.001, W=82)$. The Pearson correlation between columns 2 and 4 is: $r=84$. In our opinion, these results support the idea that the structural relation of the gray shape to the background influences the perceptual assimilation and contrast.

\section{DISCUSSION}

Many subjects reported that the lower the contrast effect, the longer the time required to see it. A reaction time experiment was therefore carried out using slides of the same patterns. Roughly the same tendency was found as in the above experiment. However, the differences between and within subjects were so large that at least 100 subjects would be required in this experiment in order to arrive at decisive conclusions.

Both the preference measure and the task could, in principle, have been oriented to the assimilation effect. However, notwithstanding the low preference measures $(\mathrm{p}<1)$ for contrast (see patterns 13, $14 \ldots$ 20 of Figure 9), there were at least two sensory factors in favor of the contrast effect: first, the midline subdividing the gray patterns (see Figure 4) enhances the contrast interpretation. This line is not specified by the codes and is therefore not accounted for in the preference measures. Second, the gray shape $\left(12 \mathrm{~cm}^{2}\right)$ covered a rather small surface area with respect to the black-white background. According to the experimental data of Helson (1963), this low gray vs. blackwhite proportion also favors the contrast effect. In any case, the contrast effect was stronger than the assimilation effect, and therefore the task was directed toward the contrast effect.

According to this analysis, assimilation and contrast effects are caused by two different interpretations of the same pattern made "simultaneously." In this respect, we do not agree with Helson's (1963) conclusion that the phenomena are complementary in nature. In our opinion, both are complementary at the effect level. The more the assimilation interpretation finds support, the more the contrast interpretation will be suppressed. For this reason, the title refers not only to contrast, but also to assimilation, although almost only contrast effects are perceived in this experiment. Notice that the lack of contrast is explained as being due to a prevalent assimilation interpretation.

At face value, a factor such as "compactness" of the gray shape seems to be related to the assimilation and contrast effect in favor of assimilation. Compactness, defined as surface divided by contour length, correlates weakly with the assimilation effect $(r=.24)$. However, compact patterns like patterns $11,12,14$, and 18 of Figure 9 show broader lines. Insofar as compactness coincides with the experimental results of Helson (1963), compactness will also indubitably be covariant with the unity of patterns. If a pattern consists of two unconnected parts, the preference measure will probably be in favor of contrast. This pattern will probably also be low in compactness. 


\section{REFERENCES}

Beck, J. Contrast and assimilation in lightness judgments. Perception \& Psychophysics, 1966, 1, 342-344.

Bezold, W. von. Die Farbenlehre im Hinblick auf Kunst und Kunstgewerbe. Braunschweig: Westermann, 1874.

Buffart, H., \& Leeuwenberg, E. Structural information theory. In H. Geissler, E. Leeuwenberg, H. Buffart, V. Sarris (Eds.), Current issues in perception. Amsterdam: NorthHolland, 1982.

Buffart, H., Leeuwenberg, E., \& Restle, F. Coding theory of visual pattern completion. Journal of Experimental Psychology, 1981, 7, 241-274.

Goldmeier, E. Similarity in visually perceived forms. Psychological Issues, Monograph, 1982, 8(1).

Helson, H. Studies of anomalous contrast and assimilation. Journal of the Optical Society of America, 1963, 53, 179-184.

Helson, H., \& JoY, V. Domains of lightness assimilation and contrast effects in vision. Psychologische Beitrage, 1962, 6, 405-415.

LEEUWENBERG, E. Quantitative specification of information in sequential patterns. Psychological Review, 1969, 76, 216-220.
Leeuwenberg, E. A perceptual coding language for visual and auditory patterns. American Journal of Psychology, 1971, 84, 307-349.

LEEUWEnBeng, E. Quantification of certain visual pattern properties: Salience, transparency, similarity. In E. Leeuwenberg \& H. Buffart (Eds.), Formal theories of visual perception. New York: Wiley, 1978.

Metelli, F. The perception of transparency. Scientific American, 1974, 230(4), 90-98.

Restle, F., \& Decker, J. Size of the Mueller-Lyer illusion as a function of its dimensions: Theory and data. Perception \& Psychophysics, 1977, 21, 489-503.

Sherif, M., \& Hovland, C. Social judgment. New Haven: Yale University Press, 1961.

Van TuiJl, H., \& Leeuwenberg, E. Neon color spreading and structural information measures. Perception \& Psychophysics, 1979, 25, 269-284.

(Manuscript received October 1, 1981; revision accepted for publication July 16, 1982.) 